\title{
Identification of Process Parameters in Electromagnetic Pulse Welding and Their Utilisation to Expand the Process Window
}

\author{
Pabst Christian and Groche Peter
}

\begin{abstract}
Impact welding, represented by the industrial processes explosion welding and electromagnetic pulse welding, offers unique advantages over conventional welding processes, especially fusion welding. The joint is produced during a high speed impact between two workpieces without additional heating or melting. Thus, even dissimilar metals can be joined. However, in contrast to fusion welding, the fundamentals are understood to a significantly lesser extent. In this publication, the effect of the density of the surrounding gaseous medium is investigated. The results show that a lower density results in a higher weld strength. At higher densities, a weld can even be inhibited completely. High speed images of the impact indicate that this is caused by the obstruction of the so-called jet due to the increased air resistance. In general, "jetting" describes the ejection of oxides, dirt and superficial material from the closing gap during the impact. It is presumed to be essential for the formation of the bond.
\end{abstract}

Index Terms - Impact welding, electromagnetic pulse welding, process window.

\section{INTRODUCTION}

The still limited understanding of the process mechanisms of impact welding makes it difficult and thus time- and money-consuming to design and optimise a joint. Besides some few empirical guidelines, barely any knowledge on the general and process-independent mechanisms and their influencing factors exists. One reason for this lack of knowledge are the high velocities during the process: The relative velocity between the workpieces to be joined is in the range of several hundred metres per second. The accelerated workpiece is usually referred to as the flyer, the stationary workpiece as the target. The impact angle between them reaches from few degrees up to $30^{\circ}$ or more. The latter is usually achieved in explosion welding only. Thus, the gap between the workpieces is closed at several thousand metres per second. Therefore, a detailed optical process observation

Manuscript received June 30, 2016; revised January 1, 2017. This work was supported by the German Research Foundation (DFG) under the grant GR 1818/49-2.

C. Pabst was with Institute for Production Engineering and Forming Machines (PtU), Technische Universität Darmstadt, Darmstadt, 64287 Germany. He is now with PSTproducts GmbH, Alzenau, 63755 Germany (e-mail: cpabst@pstproducts.com).

P. Groche is with the Institute for Production Engineering and Forming Machines (PtU), Technische Universität Darmstadt, Darmstadt, 64287 Germany (e-mail: groche@ptu.tu-darmstadt.de). is possible at some million images per second only and exposure times in the range of nanoseconds.

These velocities cause inertial effects to become significant as well. At strain rates in the range of $10^{4} 1 / \mathrm{s}$ to $10^{6} 1 / \mathrm{s}$, the density of the involved materials plays a large role. One effect in this context is the formation of the so-called jet: When the two workpieces collide at high velocities under certain angles, superficial layers are ejected out of the impact area. In literature, this phenomenon is described as dust or a solid flow of material, as shown in Fig. 1. This jet is supposed to be crucial for the formation of a bond and has a velocity in the range of several thousand metres per second. The point of the first contact between flyer and target is the collision point. Its velocity is equally in the range of several thousand metres per second and is more or less constant in explosion welding, but decreases steadily after the first contact in electromagnetic pulse welding.

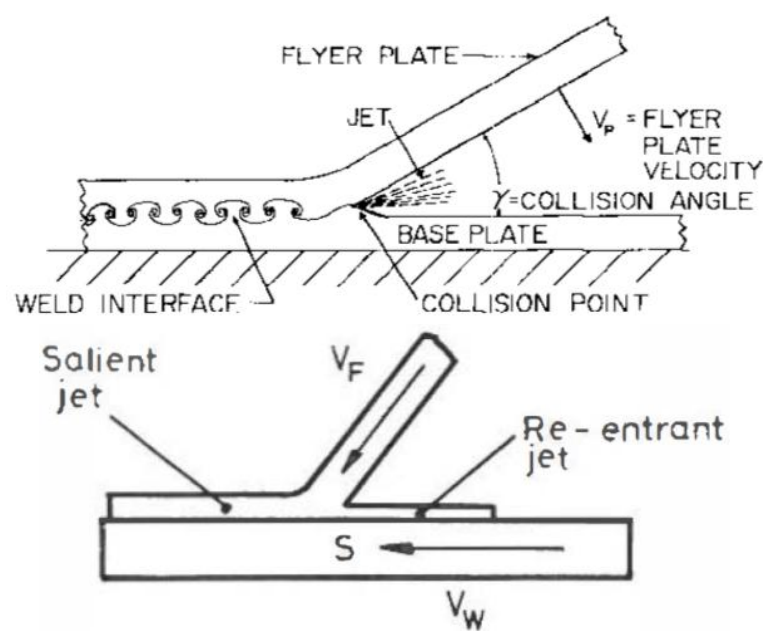

Fig. 1. Schematic depiction of the impact and the jet as spray or dust (left, [1]) and solid flow (right, [2]).

Past research works on explosion welding show that the velocity of the jet is almost twice as high in vacuum as it is at ambient pressure [3]. However, no effect on the weld quality could be found. Even the most recent works on explosion welding do not take into account the surrounding medium [4]. Previous investigations on electromagnetic pulse welding show that the width of the welded area increases significantly in vacuum and that a joint can be obtained at lower discharge currents [5]. Besides, the characteristic white flash during the impact cannot be observed in vacuum. These findings motivate the theory that the density of the surrounding medium might have an influence on the formation of the bond: The higher the density, the weaker the joint and vice versa. 
The reason could be the drag which slows down the jet and prevents it from escaping the closing gap. It stays between the workpieces and inhibits a sound joint. The velocity of the flyer might also be influenced by the density of the gas which has to be moved out of the closing gap. However, due to the difference of the flyer density and the gas density, which is around 1000, this effect will not be significant.

To study the effect of gas density, experiments with electromagnetic pulse welding under different gas atmospheres at different pressures are carried out. Besides air, the noble gases helium and argon and the inert gas sulfur hexafluoride are used.

\section{EXPERIMENTAL SETUP}

\section{A. Pulse Generator and Tool Coil}

The investigations are carried out by means of electromagnetic pulse welding. The equipment used for the trials is an industrial pulse generator "PS32-16" by PSTproducts. It has a maximum charging energy of $32 \mathrm{~kJ}$ and a maximum charging voltage of $16 \mathrm{kV}$. The sheet welding tool coil has a prismatic effective area with a cross section of $5 \times 5 \mathrm{~mm}^{2}$. The experiments are carried out with sheet metal pieces of commercially pure aluminium $(99.5 \%$ or $\mathrm{EN}$ AW-1050A) in the half hard condition $H \times 4$. The yield strength is about $77 \mathrm{~N} / \mathrm{mm}^{2}$, the tensile strength is $116-121 \mathrm{~N} / \mathrm{mm}^{2}$ with little variations due to the rolling direction. The size is $40 \times 40 \mathrm{~mm}^{2}$ and the thickness is $2 \mathrm{~mm}$. The two sheets are welded with an initial accelerating distance of $2 \mathrm{~mm}$, which is set by plastic spacers. The geometric setup is illustrated in Fig. 2. The target is backed by a steel support that is clamped to the tool coil to absorb the impulse of the impact.
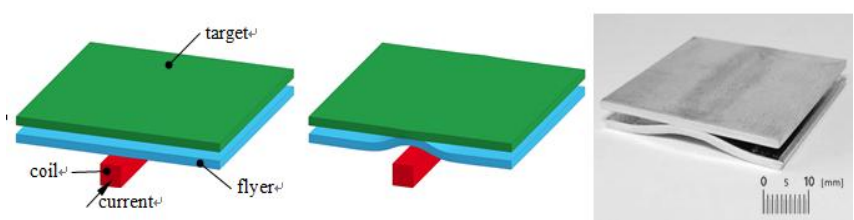

Fig. 2. Welding setup for the experiments. Left: before the welding. Middle: After the welding. Right: Photo of a welded sample.

\section{B. Process Observation}

The impact is filmed by using the image intensifier camera "hsfc pro" by PCO. It makes exposure times down to $1 \mathrm{~ns}$ and a theoretical framerate of 300 million fps possible. The number of frames is limited to four or eight, depending on the brightness of the object. Due to the process time of only few microseconds, this is not a disadvantage here. The resolution is $1208 \times 1024 \mathrm{px}$, irrespective of the frame rate. One characteristic of the impact welding process is the emission of a bright white flash during the impact. This flash can make it impossible to observe the impact area. To overcome this difficulty, the necessary light for the camera is provided by the pulsed $640 \mathrm{~nm} 400 \mathrm{~W}$ laser "CAVILUX Smart" by Cavitar. The camera is equipped with an optical $640 \mathrm{~nm}$ bandpass filter and thus it only sees the laser light, but barely the broadband process light. The camera is used to see through the $2 \mathrm{~mm}$ gap between the two sheets and the laser provides a background illumination with an additional diffusing screen. The assembly consisting of flyer, target and support is enclosed by a small acrylic glass housing. It can be evacuated to $50 \mathrm{mbar}$ and allows a certain overpressure as well. To apply different gases than air, the housing is evacuated first and then the gas is let in at ambient pressure. For the trials, the gases and pressures shown in Table I are used.

TABLE I: DESCRIPTION OF THE DIFFERENT GAS ATMOSPHERES FOR THE EXPERIMENTS

\begin{tabular}{|l|l|l|}
\hline gas & density $\left[\mathrm{kg} / \mathrm{m}^{3}\right]$ & absolute pressure [mbar] \\
\hline "vacuum" & 0.06 & 50 \\
\hline helium & 0.18 & 1030 \\
\hline ambient air & 1.20 & 1030 \\
\hline argon & 1.78 & 1030 \\
\hline ambient air & 2.40 & 2060 \\
\hline sulfur hexafluoride $\left(\mathrm{SF}_{6}\right)$ & 6.63 & 1030 \\
\hline
\end{tabular}

\section{Parameter Variation}

Two parameters are varied in the experiments: the density of the surrounding gas and the discharge energy of the pulse generator. The latter equals different discharge currents. The charging energy is varied between $10 \mathrm{~kJ}$ and $20 \mathrm{~kJ}$, which equals a charging voltage between $8.6 \mathrm{kV}$ and $12.1 \mathrm{kV}$. This leads to different peak discharge currents between $252 \mathrm{kA}$ and $356 \mathrm{kA}$ at $20 \mathrm{kHz}$ for this system. The frequency is constant, because it is mainly determined by the inductance and the capacitance of the pulse system, which consists of the pulse generator, the cables and the tool coil. The peak discharge current and the frequency are the relevant parameters for the process, because they are independent of a specific pulse system, in contrast to the charging energy and voltage. With the same peak discharge current, frequency and coil geometry, it is possible to reproduce the results with different machines. The charging energy alone is not sufficient, because other pulse generators might provide different discharge currents and frequencies at the same charging energy. The welding process is usually finished shortly after the current has reached its peak value. Table II gives an overview on the charging energy and the corresponding peak current values.

TABLE II: Discharge ENERGIES AND THE CORRESPONDING PEAK CURRENT VALUES FOR THE EXPERIMENTS

\begin{tabular}{ll|l|l|l|l|l|l|}
$\begin{array}{l}\text { charging } \\
\text { energy }\end{array}$ & $10 \mathrm{~kJ}$ & $11 \mathrm{~kJ}$ & $12 \mathrm{~kJ}$ & $14 \mathrm{~kJ}$ & $16 \mathrm{~kJ}$ & $18 \mathrm{~kJ}$ & $20 \mathrm{~kJ}$ \\
\hline $\begin{array}{l}\text { peak } \\
\text { current }\end{array}$ & $\begin{array}{l}252 \\
\mathrm{kA}\end{array}$ & $\begin{array}{l}263 \\
\mathrm{kA}\end{array}$ & $\begin{array}{l}275 \\
\mathrm{kA}\end{array}$ & $\begin{array}{l}297 \\
\mathrm{kA}\end{array}$ & $\begin{array}{l}318 \\
\mathrm{kA}\end{array}$ & $\begin{array}{l}338 \\
\mathrm{kA}\end{array}$ & $\begin{array}{l}357 \\
\mathrm{kA}\end{array}$ \\
\hline
\end{tabular}

\section{Evaluation of the High Speed Images}

Fig. 3 shows an exemplary high speed image from the process and the same state from a numerical simulation. The image is taken $20.75 \mu \mathrm{s}$ after triggering the switches of the pulse generator. The jet, evolving from the impact area, can be clearly identified. Due to the perspective of the camera and the optical path through the gap, reflections of the jet can be seen at both the target above and the flyer below. 


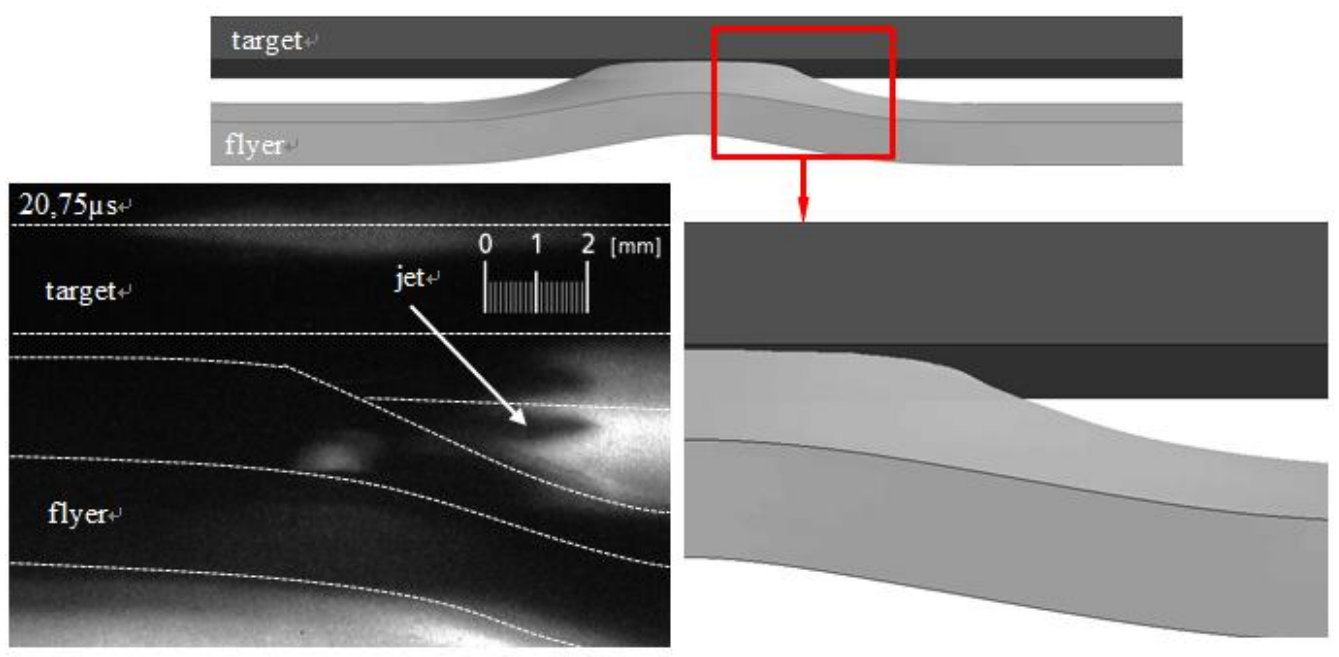

Fig. 3. Description of the camera image (left) with the help of results from a numerical simulation.
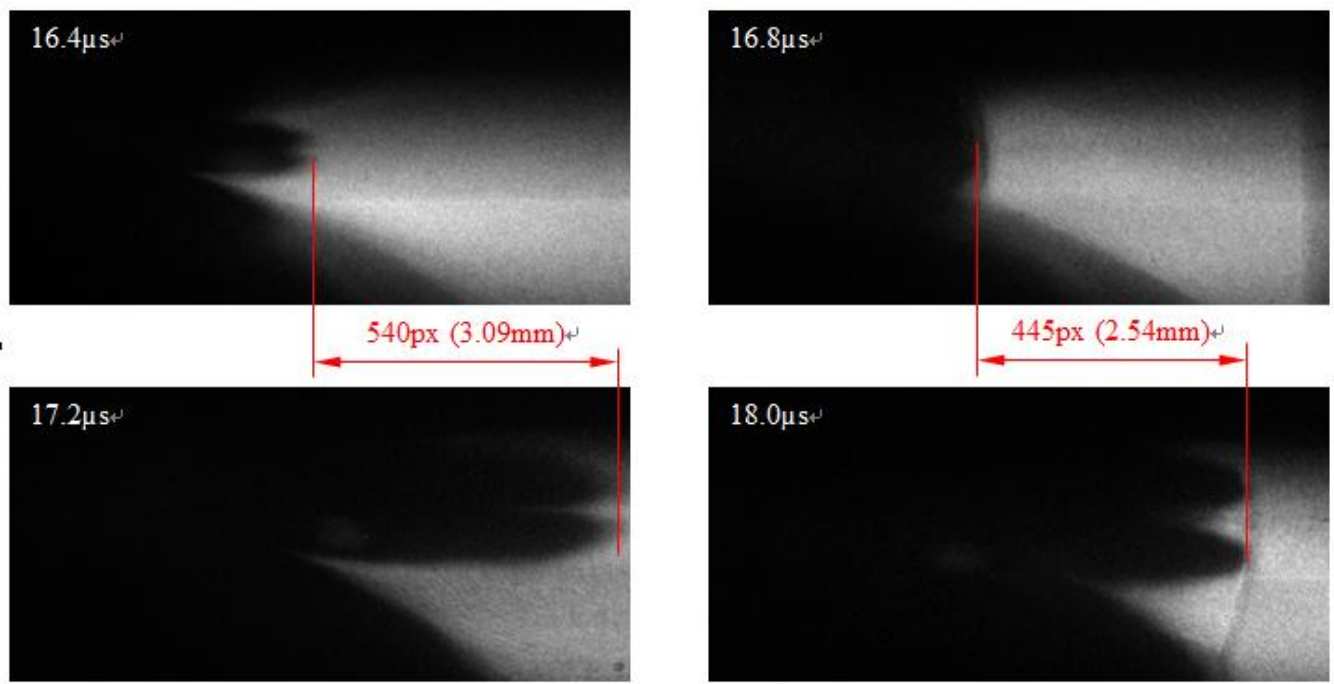

Fig. 4. Examples of the jet velocity measurement. Left: $12 \mathrm{~kJ}$ in air at ambient pressure. The shock wave is visible. Right: $12 \mathrm{~kJ}$ in vacuum. Note the different time steps between the images.
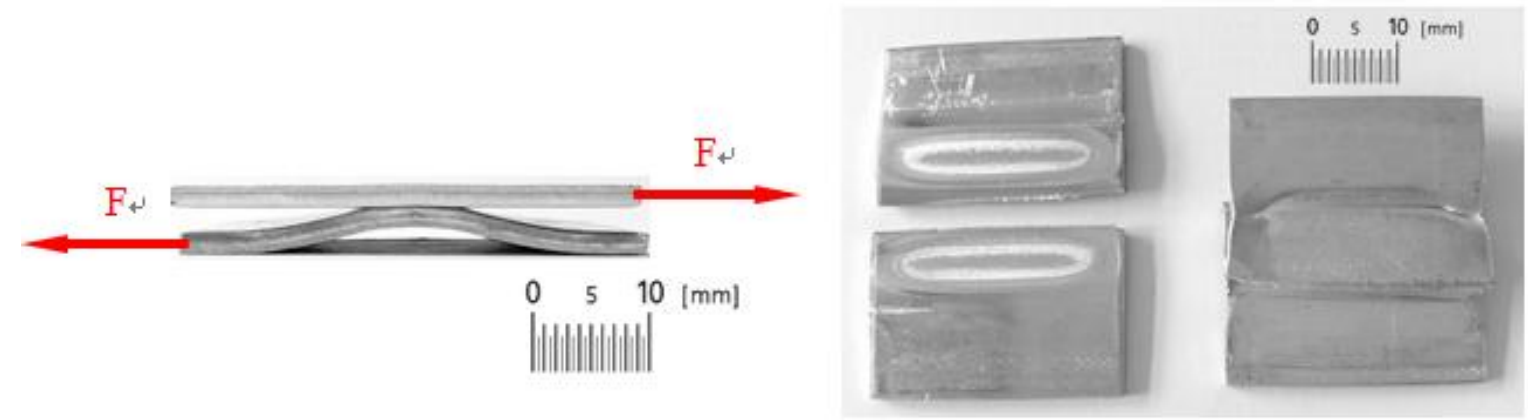

Fig. 5. Left: Tensile test setup. Right: Tested samples with failure of the weld and outside the weld in the base material.

The velocity of the jet is acquired by comparing two images from the sequence and measuring the distance covered by the jet. In Fig. 4, 175 \pm 5 px equal $1 \mathrm{~mm}$. With a delay of $1.2 \mu \mathrm{s}$ and $0.8 \mu$ s between the images, this equals an average jet velocity of $2120 \mathrm{~m} / \mathrm{s}$ and $3860 \mathrm{~m} / \mathrm{s}$, respectively. It must be noted that the velocity of the jet decreases steadily during the impact. To minimize the scattering, the velocities are always measured at approximately the same position.

\section{E. Tensile Tests}

Selected welded samples are shear tested afterwards. The setup is illustrated in Fig. 5 (left). The theoretical maximum strength of about $9480 \mathrm{~N}$ is limited by the base material. However, this strength cannot be reached. One reason is that the flyer is subjected to macroscopic deformations. In addition, the welded area is o-shaped (Fig. 5) and not homogeneous across the width of the sheets. Thus, stress concentrations exist at the edges and reduce the overall strength of the joint. Therefore, a sample with a good weld fails close to the weld in the base material (Fig. 5, right).

\section{RESULTS}

Fig. 6 shows the jet velocity depending on the charging 
energy and the density. Only successful welds are shown here and it can be seen that no weld is possible below about $2000 \mathrm{~m} / \mathrm{s}$. With sulfur hexafluoride $\left(6.63 \mathrm{~kg} / \mathrm{m}^{3}\right)$, no joint was possible even at $16 \mathrm{~kJ}$ charging energy. The measured jet velocity was only about $1800 \mathrm{~m} / \mathrm{s}$ in this case. The jet velocity increases with increasing charging energy and with decreasing ambient gas density. However, a stronger correlation between jet velocity and charging energy was expected.

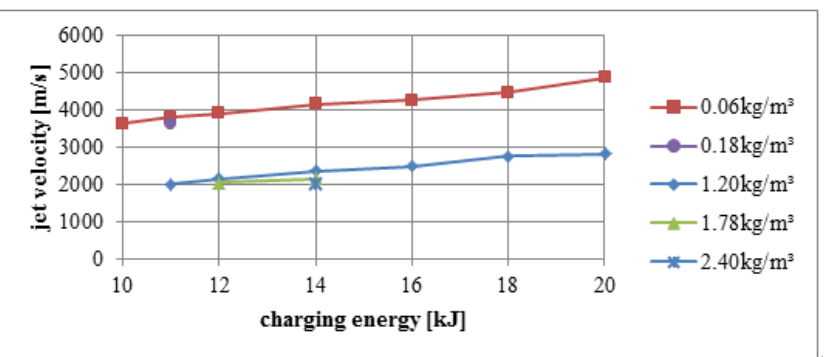

Fig. 6. Jet velocity vs. charging energy for different ambient gas densities. Note that the values for successful bonds are given only.

Fig. 7 shows the theoretical maximum tensile force for a sample $(9480 \mathrm{~N})$ and the measured forces for different ambient gas densities depending on the charging energy. It can be seen that the density has a large effect on the tensile force at low charging energies. This effect disappears at higher energies $(18 \mathrm{~kJ})$ when the theoretical maximum force is almost reached. After the peak, the tensile force slightly decreases. This can be caused by shock effects especially in the target sheet which weaken the material due to microscopic cracks. The samples still fail close to the joint area.

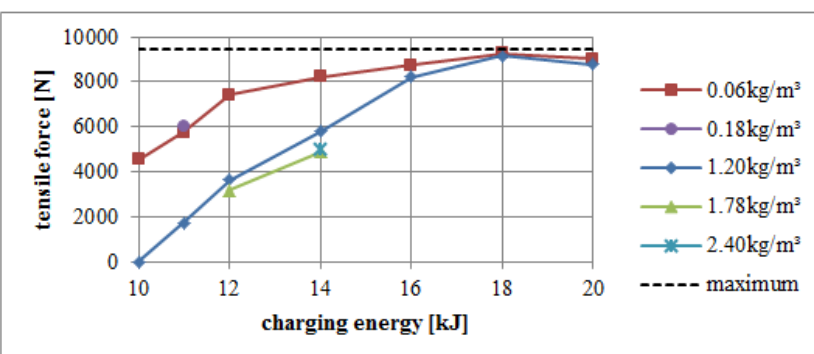

Fig. 7. Tensile force vs. charging energy for different ambient gas densities and the theoretical maximum strength.

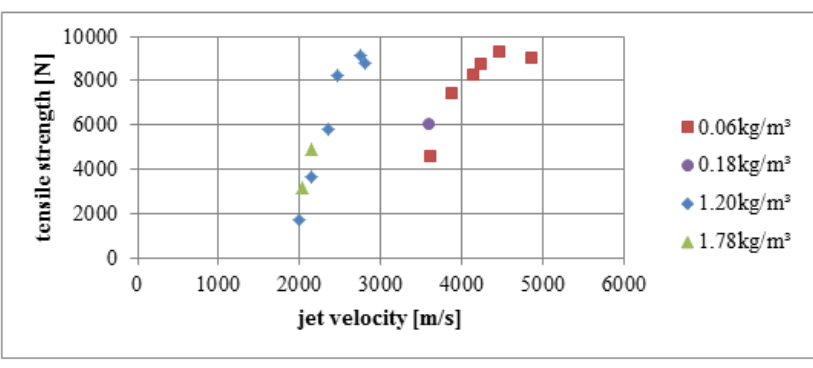

Fig. 8. Tensile force vs. jet velocity for different ambient gas densities.

Fig. 8 shows the strength of the joint depending on the jet velocity for four different ambient gas densities. It can be concluded that the tensile force strongly depends on the jet velocity. It is obvious that a certain jet velocity cannot be correlated with certain bond strength without taking into account the density of the surrounding gas atmosphere. When the peak strength is reached, a further increase is not possible and the measuring points continue moving to the right to higher jet velocities.

\section{DISCUSSION AND CONCLUSION}

The results shown in Fig. 8 support the theory that the strength of the bond correlates with the velocity of the jet. It was not possible to obtain even a weak joint below a jet velocity of about $2000 \mathrm{~m} / \mathrm{s}$ with none of the gases and pressures, as shown in Fig. 6. However, it is not possible to predict the strength of the joint merely by the jet velocity, which can be seen in Fig. 8. The density of the atmosphere seems to play another large, unknown role and appears to not only influence the jet velocity. One explanation might be the transient behaviour of electromagnetic pulse welding. The jet velocity decreases during the process because the collision point velocity decreases as well. As it was not possible to observe the process in close vicinity to the first contact between flyer and target, where much higher velocities are expected to occur, other mechanisms might be active there.

Another, analytical explanation can be given regarding the energy budget. The kinetic energy of the flyer, which is provided by the pulse generator and transferred by the tool coil, is used to form the weld. A part of this energy has to be used to remove the particles from the bonding area, this is the jet. This energy is transferred partially to the particles and partially to the ambient gas that has to be moved as well. A higher kinetic energy for the jet can be achieved by increasing the charging energy or by reducing the density and thus the mass of the ambient gas. A higher kinetic energy, however, does not necessarily correspond to higher velocity. A larger jet with identical velocity is also possible. In fact, the jet is thinner in vacuum than in other gases, which can be clearly seen in Fig. 4. In contrast to the velocity, the kinetic energy cannot be measured so far. Even the measurement of the jet's size in the high speed images as a proportional value, under the assumption that the density is always constant, is not possible so far with an acceptable accuracy.

As shown in Fig. 7, welds in vacuum occur earlier and reach higher strengths at lower energies than in air at ambient pressure. However, surprisingly, the maximum strength at which the base material fails is reached at the same charging energy. Above this value, tensile tests do not provide valuable information, because an increasing weld area cannot be identified when the base material fails. The aim in the industrial application is always to gain a sound weld, which would be the area around $18 \mathrm{~kJ}$ in Fig. 7. However, a decreased ambient pressure or helium do not provide any advantage there. Nevertheless, challenging material combinations might profit from a helium or vacuum atmosphere and reach larger weld areas and higher strengths.

Another conclusion can be drawn from the results: It seems as if there is no difference whether the surrounding gas is inert or not, whereas the density is the governing property.

\section{SUMMARY}

Electromagnetic pulse welding trials under different gases and pressures have been carried out. It was shown that the process window can be extended to lower discharge energies 
if the weld is produced under an atmosphere with a lower density than ambient air. Measurements of the jet velocity reveal that it correlates directly with the ambient gas density. For the same gas atmosphere, the weld strength correlates with the jet velocity. However, the measured jet velocity from the experiments cannot be used to predict the weld quality without taking the ambient density into account.

\section{OUTLOOK ON FURTHER INVESTIGATIONS}

One aspect has not been taken into consideration so far: At different charging energies, the macroscopic deformation of the flyer will not only be faster, but also slightly different. This changes both the impact angle and the collision point velocity. However, this can be hardly observed with the camera so far. Current numerical simulations allow a rough estimate only. Due to the high strain rates and the resulting complex material modelling, large deviations can occur [6].

Another factor might be the thermal behaviour of the gases during the rapid compression inside the closing gap. It is suggested that temperatures might reach more than $1000{ }^{\circ} \mathrm{C}$ in this gap due to the supersonic compression [7]. This might also enable chemical reactions with oxygen.

In future experiments, the testing will also be improved. The tensile tests will be carried out with samples where the outer edges that cause the stress concentrations are cut off. 3D ultrasonic testing is planned to determine the welded area and its shape. This is particularly important for those samples that do not break in the weld area. It is assumed that the tensile strength of the sample is directly proportional to the size of the weld, which again is directly proportional to the charging energy or the discharge current. However, at high tensile forces, the influence of the base material and the non-uniformity of the stress distribution introduce nonlinearity, which can be seen in Fig. 7. Therefore, the actual size of the welded area is a more suitable and reliable quantity to describe the weld's properties than the tensile force of the sample.

\section{ACKNOWLEDGEMENTS}

The authors would like to thank PCO and Acal BFi for their support in developing the imaging system. The authors also appreciate the support by PSTproducts in providing the pulse generator and the tool coil for the trials.

\section{REFERENCES}

[1] S. H. Carpenter and R. H. Wittman, "Explosion welding," Annu. Rev. Mater. Sci., vol. 5, pp. 177-199, 1975.

[2] B. Crosslan, Explosive welding of metals and its application. Oxford Series on Advanced Manufacturing, Oxford University Press, Oxford, 1982.

[3] O. R. Bergmann, G. R. Cowan, and A. H. Holtzman, "Experimental evidence of jet formation during explosion cladding," Trans. Metall. Soc. AIME, vol. 236, pp. 646-653, 1966.

[4] V. I. Lysak, S. V. Kuzmin, "Lower boundary in metal explosive welding. Evolution of ideas," J. Mater. Process Technol., vol. 212, pp. 150-156, 2012.

[5] C. Pabst and P. Groche, "Electromagnetic pulse welding: Process insights by high speed imaging and numerical simulation," in Proc. 6th ICHSF, 2014, pp. 77-88.

[6] P. Groche and C. Pabst, "Numerical simulation of impact welding processes with LS-DYNA," in Proc. 10th European LS-DYNA Conference, 2015

[7] M. Koschlig, M. Veehmayer, and D. Raabe, "Production of steel-light metal compounds with explosive metal cladding," in Proc. 3rd ICHSF, 2008, pp. 23-32.

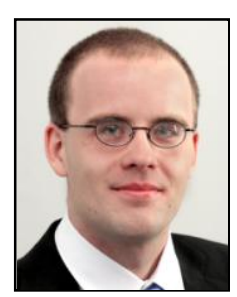

C. Pabst was born in Berlin in 1987. After achieving his master's degree in mechanical and process engineering at the University in Darmstadt in 2011, he worked as PhD student at the Institute for Production Engineering and Forming Machines (PtU) for five years. Since October 2016, he is development engineer at PSTproducts $\mathrm{GmbH}$.

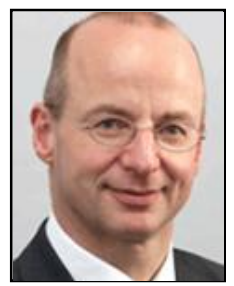

P. Groche was born in Marl in 1961. After his studies of Mechanical Engineering at the Technical University of Braunschweig in 1986, he worked as a research associate at the Institute of Metal Forming and Forming Machines at the University of Hanover. After his doctorate in 1990, he worked in the industry until 1999 when he became the director of the Institute for Production Engineering and Forming Machines. 